\title{
The market demand- (and supply) curve paradox
}

\author{
Kjetil K. Haugen** Knut P. Heen \\ Faculty of Business Administration and Social Sciences, Molde University College, \\ Specialized University in Logistics, Molde, Norway
}

Received: 19 February 2020

Revised: 26 May 2020

Accepted: 17 June 2020

\begin{abstract}
After many years of teaching utility maximization in Microeconomics a certain paradoxical puzzle has come to our attention. It is very simple and straightforward, but we still find it hard to explain it to students. Our hope is that the distinguished community of theoretical economists may help us solve this mystery. After all, we would find it extremely unlikely that we are the first persons to identify this paradox.

Keywords: micro economics; utility maximization; demand curve; market demand curve; supply curve

JEL Classification Codes: A11, D11, Q11
\end{abstract}

\section{Introduction}

Demand curves are central in almost all economic theory. Such curves define a causality between price and quantity in a market, and they are essential (as input) to most economic analysis. Classical microeconomic theory ${ }^{1}$ tells a story on how demand curves can be derived based on utility maximization.

The story continues as follows: We start with a single consumer and solve his/her's utility maximization problem for given prices (on two products to make it simple), income and preferences. This solution contains optimal amounts for this consumer's choice of the two products, as well as accompanying prices say $\left(X_{1}, Y_{1}\right)$ and $\left(p_{X}^{1}, p_{Y}^{1}\right)$ Now, a change in the price of one of the goods, say $p_{X}$, from $p_{X}^{1}$ to , $p_{X}^{2}$ would induce a new utility maximization problem, with a new solution, say $\left(X_{2}, Y_{2}\right)$ and prices $\left(p_{X}^{2}, p_{Y}^{1}\right)$. Repeating this "algorithm" leads to two vectors $\boldsymbol{X}=\left[X_{1}, X_{2}, \cdots\right]$ and $\boldsymbol{P}=\left[p_{X}^{1}, p_{X}^{2} \cdots\right]$ which together define the demand curve (for $\boldsymbol{X}$ ) for this consumer. Repeating this for all (say $N$ ) consumers in the market will hence produce individual demand curves for each consumer and a straightforward aggregation technique will produce the final result - the market demand curve.

\footnotetext{
* Corresponding author. E-mail: Kjetil.Haugen@ himolde.no.

Citation: Haugen, K. K., and Heen, K. P. (2021) The market demand- (and supply) curve paradox, Economics and Business Letters, 10(1), 69-71.

DOI: 10.17811/ebl.10.1.2021.69-71

${ }^{1}$ Refer to any standard textbook in Microeconomics, for instance Pindyck and Rubinfeld (2018), The argument presented here is typically omitted in most textbooks, also this one.
} 


\section{The paradox}

$$
\operatorname{Max}_{X, Y}\left\{U(X, Y) \mid p_{X} X+p_{Y} Y \leq I\right\}
$$

The optimization problem (1) is one among many potential descriptions of a two-good utility maximization problem. It contains 4 bits of input; the prices of each good, $\left(p_{X}, p_{Y}\right)$, the income of the consumer, $I$, as well as the consumer's preferences $U()$. These inputs need to be taken as given (at least parametrically) in order to solve the problem ${ }^{2}$.

So far, no problems. However, when solving the problem (1), some notion on potential other consumers in the economy must be made for such a utility maximization to make sense. After all, if the consumer acts in a "small" economy he or she may be tempted to start negotiating the price. In such a case, of course, the price is not given. Hence, some size of the economy is needed for a meaningful solution of problem (1). One typical argument is to state that the economy contains so many consumers that the choice of a single agent will not affect equilibrium prices.

Unfortunately, this does not solve the problem, as any finite sized consumer set can open for coalitions, especially these days with social media as an obvious channel to establish such. In fact, the only valid necessary assumption for a meaningful solution of (1) is an infinite number of consumers. In such a situation, any finite coalition is (fortunately) infinitely small compared to the remaining infinite number of consumers. That is:

Assumption 1: $N=\infty$.

Now, we happily move on with the teaching story described in section 1. Everything goes well, until the very final step - the aggregation. The problem is obvious, isn't it? The aggregation step involves using all the information gathered so far and starts by picking some price. For this price, say $\hat{p}_{X}$, identify all accompanying demands for all consumers and add all these demands together. This number (obviously) is the total market demand for the given $\hat{p}_{X}$. In most textbooks (again Pindyck and Rubinfeld (2018) may serve as a reference), the number of consumers is 3 or maybe 4. But at this point, the explicit or implicit Assumption 1 is totally forgotten. If we start out with an infinite number of consumers, then we have to aggregate the same infinite number of consumers when the market demand curve is found. And, here is the problem. As $\sum_{i=1}^{\infty} d_{i} \mid d_{i} \geq 0=\infty^{34}$, no sensible market demand curve exists.

At this point, the concept of 'price taking behavior' may be suitable to address. Many microeconomic textbooks use this term as an alternative to Assumption 1. As we see it, this does not really change anything. After all, the idea is the same. Price taking behavior is only defendable if the individual consumer is so small compared to the whole set of consumers that his/her's consumption decision does not have any impact on commodity price. And, as argued above, the only waterproof logic to secure no possibility of coalition formation is an infinite amount of consumers.

In Pindyck and Rubinfeld (2018), the 'price taking' concept is introduced in production theory. It ought to be obvious, that this (in our terms) corresponds to an infinite number of producers, and hence a similar argument for non-existence of the supply curve as for the demand curve.

That is, not only can existence of demand curves, but also supply curves be questioned. An obvious consequence is of course that the perfectly competitive equilibrium model itself is under attack.

\footnotetext{
${ }^{2}$ Economists often refer to the input as being exogenously given.

${ }^{3} d_{i}$ denotes demand from consumer $i$.

${ }^{4}$ Of course, the special case $d_{i}=0 \forall i$, works, but it is unfortunately the only working case. And, it does not really help. As long as there is an infinite number of demands $\left(d_{i}\right.$ 's), and if not all are zero, the problem remains. Or, put another way, if there is a certain finite positive probability that any $d_{i}>0$, the sum turns to equal infinity.
} 


\section{Conclusions}

Fortunately, our experience has shown that few if any students remember Assumption 1 when the aggregation step is reached. Normally most students (in a basic course) wonder much more about what a utility function means, the concept of mathematical programming, Lagrange multiplicators and so on. Hence, none of the hundreds or even thousands of students passing through our classrooms over the years have ever identified this paradox.

However, at some point, somebody will. Then, the question arises, how should we answer? We will probably, use the classical "all models are in reality approximations". As always, when a situation demands more understanding than the teacher has, this trick is applicable. Still, this paradox makes us wonder: How could a building (economic theory) built on such a fragile foundation (market demand curves) make sense? After all, how can an argument starting out with the assumption of an infinite $\mathrm{N}$, ending up with a finite $\mathrm{N}$ make sense for anybody, teachers or students?

\section{References}

Pindyck, R., and Rubinfeld, D. (2018) Microeconomics, Pearson, 9th edition. 